\title{
Tonsillobiventral fissure approach to the lateral recess of the fourth ventricle
}

\author{
Ali Tayebi Meybodi, MD, Michael T. Lawton, MD, Halima Tabani, MD, and Arnau Benet, MD \\ Skull Base and Cerebrovascular Laboratory, Department of Neurosurgery, University of California, San Francisco, California
}

OBJECTIVE Surgical access to the lateral recess of the fourth ventricle (LR) is suboptimal with existing transvermian and telovelar approaches because of limited lateral exposure, significant retraction of the cerebellar tonsil, and steep trajectories near brainstem perforator arteries. The goal in this study was to assess surgical exposure of the tonsillobiventral fissure approach to the $L R$, and to describe the relevant anatomy.

METHODS Two formaldehyde-fixed cerebella were used to study the anatomical relationships of the LR. Also, the tonsillobiventral fissure approach was simulated in 8 specimens through a lateral suboccipital craniotomy.

RESULTS The pattern of the cerebellar folia and the cortical branches of the posterior inferior cerebellar artery were key landmarks to identifying the tonsillobiventral fissure. Splitting the tonsillobiventral fissure allowed a direct and safe surgical trajectory to the LR and into the cerebellopontine cistern. The proposed approach reduces cervical flexion and optimizes the surgical angle of attack.

CONCLUSIONS The tonsillobiventral fissure approach is a feasible and effective option for exposing the LR. This approach has more favorable trajectories and positions for the patient and the surgeon, and it should be added to the armamentarium for lesions in this location.

https://thejns.org/doi/abs/10.3171/2016.8.JNS16855

KEY WORDS cerebellar tonsil; telovelar approach; cerebellomedullary fissure; inferior medullary velum; biventral lobule; tela choroidea; cerebellopontine cistern; vascular disorders; anatomy

$\mathrm{T}$ HE lateral recess of the fourth ventricle (LR) is a narrow space bounded by vital neural and vascular structures, which make surgical access to this region difficult. ${ }^{4}$ As first described, this area was accessed by splitting the vermis and opening the foramen of Magendie (transvermian approach), which required significant retraction of the cerebellar hemispheres. ${ }^{2,15-17}$ The transvermian approach provides limited exposure of the LR and is associated with risk of cerebellar mutism, especially in the pediatric population. ${ }^{3,12,19}$ An alternative approach is the transcerebellomedullary fissure (tCMF) approach, also known as the telovelar approach., $, 6,10,17$ This approach requires opening the cerebellomedullary fissure (CMF) and the telovelar membrane (a thin membrane forming the lower portion of the roof of the fourth ventricle) to reach the LR. ${ }^{10}$ The tonsil is retracted laterally and away from the brainstem, but exposure of the most lateral extent of the LR (near the foramen of Luschka) is difficult, ${ }^{4}$ despite opening the uvulotonsillar fissure to release cerebellar tension. ${ }^{8,11}$ Both transvermian and tCMF approaches to the LR provide an angle of attack almost parallel to the axis of the brainstem, requiring significant neck flexion during patient positioning and creating an uncomfortable position for the surgeon. The steep angle of attack reduces maneuverability and access to the LR. The C-1 lamina blocks the line of sight to the CMF, necessitating a laminectomy. A third option for exposing the LR is tonsillar resection. ${ }^{4}$ Although resection of the tonsils has not been associated with loss of function, it is more invasive than the other approaches and could cause damage to the inferior cerebellar peduncle. ${ }^{4}$

Splitting the tonsillobiventral fissure was previously introduced by our team to address lesions of the inferior cerebellar peduncle. This tonsillobiventral fissure approach, also more simply known as the supratonsillar approach, has a superolateral trajectory over the cerebellar tonsil leading to the inferior cerebellar peduncle that is inferior and lateral to the dentate nucleus. In our previous work, we demonstrated that this supratonsillar approach was ideal for removing peduncular cavernous malformations while minimizing transgression of normal cerebellar tissue. In the present work, we used the same surgical corridor but examined its access to the LR by using a different trajectory, with the goal of providing anatomical landmarks for its clinical application. 


\section{Methods}

Two extracted brain specimens were prepared with standard embalming solution to study the regional anatomy of the LR and design the surgical approach. In addition, 4 human heads ( 8 specimens) were prepared for surgical simulation at the University of California, San Francisco's skull base and cerebrovascular laboratory, using our previously reported method. ${ }^{1}$ The tonsillobiventral fissure approach and the tCMF approach to the LR were performed as previously reported elsewhere. ${ }^{717}$ A photographic report was performed to depict the surgical trajectory and key anatomical landmarks. A descriptive comparative analysis, including a description of the limits of the surgical exposure, surgical corridor and trajectory, and vessels at risk was undertaken to assess the surgical feasibility of the tonsillobiventral fissure approach to the LR.

\section{Results}

\section{Anatomical Relationships of the LR}

The LR is a triangular space in the lateral corner of the rhomboid fossa (Fig. 1A). It is limited ventrally by the brainstem and dorsally by the cerebellum. The inferior cerebellar peduncle sits in the lateral aspect of the dorsal surface of the medulla. It forms the ventral wall and roof of the LR, merging dorsally into the cerebellum (Fig. 1A and B). The dorsal wall and floor of the LR are formed by the lateral continuation of the tela choroidea and the inferior medullary velum (the telovelar membrane). The tela choroidea is a thin semitransparent layer of tissue from which the choroid plexus protrudes into the fourth ventricle (Fig. 1C). The tela is attached to the teniae-an elevated ridge along the inferolateral borders of the rhomboid fossa. The dorsal surface of the fourth ventricle (also referred to as the caudal half of the roof of the fourth ventricle) is formed by the tela caudally, and the inferior medullary velum cranially. The inferior medullary velum-a thin layer of neural tissue-connects the nodule to the flocculus, which protrudes into the cerebellopontine cistern through the foramen of Luschka (Fig. 1C). The horizontal arm of the choroid plexus is attached to the tela and spans the length of the LR from medial to lateral, until it emerges through the foramen of Luschka in the cerebellopontine cistern. The CMF runs between the medulla and the cerebellar tonsil and reaches the LR superiorly (Fig. 1D and E).

The cerebellar tonsil is attached to the rest of the cerebellar tissue through a peduncle, located superolaterally. Anteriorly, the tonsil is separated from the medulla by the telovelar membrane (Fig. 1C). From the tCMF approach, the LR may be accessed after opening the CMF, which is accomplished by elevating the anterior surface of the tonsil from the dorsal surface of the medulla and dividing the tela (Fig. 1D and E).

The tonsil is related superolaterally to a portion of the cerebellar hemisphere called the biventral lobule. The fissure between the tonsil and the biventral lobule is called the tonsillobiventral fissure. This fissure runs inferiorly and laterally from the valley between the tonsil and the vermis to the lateral part of the CMF (Fig. 1F). When split and followed caudally, the tonsillobiventral fissure opens into the LR (Fig. 1G). The CMF is continuous with the tonsillobiventral fisure around the superior pole of the tonsil. The posterior inferior cerebellar artery (PICA) emerges from this fissure to continue its cortical course $\left(\mathrm{P}_{4}-\mathrm{P}_{5}\right.$ transition $^{14}$ ) (Fig. 1F).

\section{Tonsillobiventral Fissure Approach to the LR}

Access to the LR was achieved by splitting the tonsillobiventral fissure (Fig. 1G). After completion of a lateral suboccipital craniotomy and opening of the dura mater, the tonsillobiventral fissure was recognized and opened. Key landmarks for efficient exposure of the tonsillobiventral fissure were the vertical direction of the tonsillar folia relative to the folia of the biventral lobule (coursing diagonally) as well as the emergence of at least 1 major branch of the PICA. The dorsal and cranial aspects of the LR were exposed after continued microsurgical arachnoid dissection in an inferolateral trajectory. The contents of the LR, namely the telovelar membrane, the choroid plexus, and the rhomboid lip covering the choroid plexus, were accessed via the tonsillobiventral fissure approach (Fig. 1G). During opening of the tonsillobiventral fissure to reach the LR, no traversing of neural tissue was required and arachnoid dissection in an inferolateral trajectory led to the LR.

Because of the anatomical features of the tonsillobiventral fissure, the surgical trajectory of the tonsillobiventral approach was almost perpendicular to the axis of the brainstem (Fig. 2). Also, this approach enabled exposure of the most lateral extent of the LR all the way to the cerebellopontine cistern without transgressing neural tissue (Fig. 1G).

Since we published our report on the supratonsillar approach, ${ }^{7}$ we have operated on 9 cases of cavernous malformations of the cerebellum, using the tonsillobiventral fissure as the sole surgical corridor. Our anatomical research as well as clinical experience with the tonsillobiventral fissure inspired its use for LR access. Although our clinical applications are limited to cerebrovascular lesionsand cavernous malformations in particular-due to the subspecialty focus of the senior author (M.T.L.), the large exposure and optimal trajectory to the LR provided by the tonsillobiventral fissure approach justify its use for tumors as well. Choroid plexus tumors, ependymomas, neuromas of the caudal cranial nerves, and epidermoid tumors infiltrating the LR can also be addressed via this approach.

\section{Case Illustration}

A 24-year-old man presented with headaches related to heavy weight lifting. An MRI study of the brain revealed a lesion in the right LR that raised our concern for cavernous malformation. After discussing treatment options, the patient decided to pursue resection. A tonsillobiventral approach was performed and the lesion was resected en bloc (Fig. 3). The patient remained symptom free 3 months after the operation.

\section{Discussion}

This report shows that the tonsillobiventral fissure approach provides an optimal trajectory to the LR through the tonsillobiventral fissure. We observed and identified several 

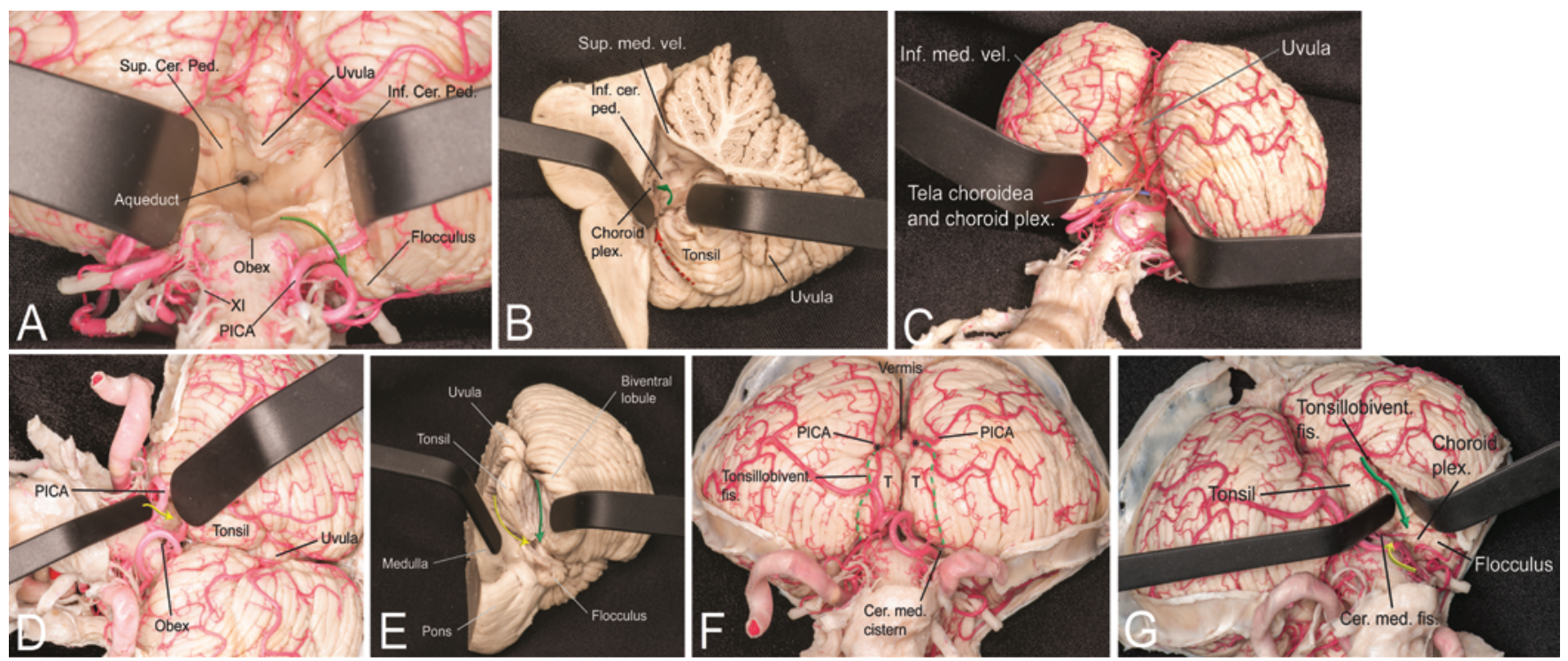

FIG. 1. A: The fourth ventricle and rhomboid fossa viewed from an inferior trajectory. The tonsils are resected. The LR is located at the lateral corners of the rhomboid fossa. When followed laterally, it will open into the cerebellopontine cistern. The right LR is shown with a curved green arrow. Note the close relationship between the inferior cerebellar peduncle and the LR. B: Lateral view of the cavity of the fourth ventricle and the LR of the right half of the brainstem and cerebellum. The inferior cerebellar peduncle curves over the LR (green arrow) to connect the medulla oblongata to the cerebellum. The tonsillobiventral fissure (red dashed arrow) reaches the LR. C: Oblique view of the lower brainstem and cerebellum with the tonsils removed. The tela choroidea and the choroid plexus of the fourth ventricle are shown. The tela and the inferior medullary velum form the lower half of the roof of the fourth ventricle. The tela forms the posterior aspect of the LR. Blue probe shows the cavity and trajectory of the LR. D: The $\mathrm{CMF}$ is opened by elevation of the tonsil. This maneuver will expose the LR (yellow arrow). E: The tonsillobiventral fissure (green arrow) is opened in another specimen viewed from below. It is clear that the opening of the tonsillobiventral fissure leads to the LR. Please compare the different trajectories of the 2 approaches. F: The inferior view of the brainstem and the cerebellum. Note the relationship of tonsil to the biventral lobule and the vermis. The tonsillobiventral fissure (dashed green lines) runs inferolaterally from the uvulotonsillar junction to reach the cerebellomedullary cistern. The PICA emerges from the tonsillobiventral fissure to branch onto the suboccipital surface of the cerebellum, as denoted by the asterisks. G: Tonsillobiventral fissure approach. The right tonsillobiventral fissure is split to show the inferolateral trajectory to the LR of the fourth ventricle (green arrow). Note the exposed choroid plexus and the flocculus in the cerebellopontine cistern. Yellow arrow shows the trajectory of the tCMF approach to the LR. Cer. = cerebellar; fis. = fissure; Inf. = inferior; med. = medullary; Ped. = peduncle; plex. $=$ plexus; $\mathrm{T}$ = tonsil; Tonsillobivent. $=$ tonsillobiventral; Sup. = superior; vel. = velum; $X I=11$ th cranial nerve. Figure is available in color online only.

advantages of the proposed approach compared with its alternatives, the tCMF and transvermian approaches.

One of the major advantages of the tonsillobiventral approach is its ergonomic angle of attack, which allows a more comfortable operation for the surgeon and safer positioning for the patient. The tonsillobiventral fissure has a vertical trajectory relative to the brainstem compared with the CMF (Fig. 2). This anatomical feature facilitates surgical access to the LR by lessening the need for hyperflexion of the patient's head when in the prone position, and by eliminating the need for a C-1 laminectomy. In the transvermian and $\mathrm{tCMF}$ approaches, the angle of attack is almost parallel to the brainstem axis and requires maximal neck flexion. Also, in such approaches, the steep angle of attack causes the cervical musculature and the C-1 lamina to obstruct the line of sight to the LR. ${ }^{2}$ These problems are virtually absent when the LR is accessed through the tonsillobiventral fissure. A slight rotation of the patient's head toward the side of the lesion brings the tonsillobiventral fissure into the line of sight of the surgeon and improves surgical trajectory.

The tonsillobiventral approach requires minimal cerebellar retraction to reach the LR. The tonsillobiventral fissure leads directly to the LR (Fig. 1D, E, and G). During this approach, the tonsil is gently mobilized inferomedially, while the biventral lobule is retracted superolaterally (tonsillobiventral fissure split). The relatively vertical approach to the LR, and the reduced retraction of cerebellar tissue, both allow maximal protection of the neural tissue, which might minimize the risk of cerebellar mutism after this approach. ${ }^{17}$

Although it shares a common surgical corridor, the tonsillobiventral fissure approach to the LR is different from the one in our previous report on the supratonsillar approach to the inferior cerebellar peduncle in several ways. ${ }^{7}$ Because the inferior cerebellar peduncle sits superior to the LR, the trajectories to these 2 targets are different: inferolateral for the LR, and superolateral for the inferior cerebellar peduncle. As described in our previous work, accessing the inferior cerebellar peduncle invariably requires a far-lateral approach, in which the head is turned contralateral to the lesion and the neck is flexed. This position aligns the surgical view to the inferior cerebellar peduncle along the axis of the tonsillar peduncle? On the other hand, the LR can be accessed following an inferolateral trajectory, and the prone position with modest neck flexion is sufficient to align the LR with the surgical view. The extreme neck flexion and the three-quarter 

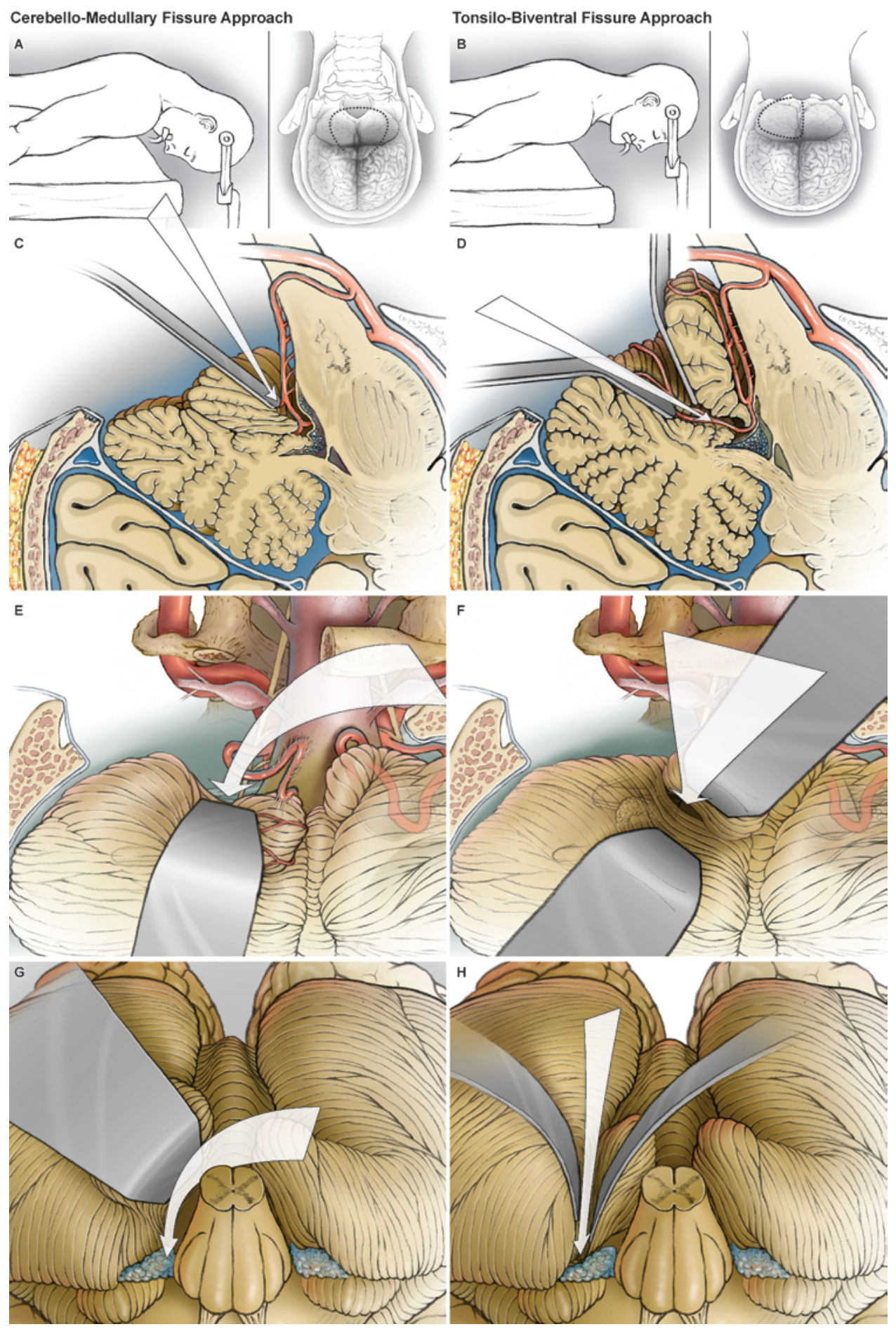

FIG. 2. Schematic illustration and comparison of the CMF and tonsillobiventral fissure approaches to the LR. A and B: Patient positioning and craniotomy. C and D: Sagittal trajectory and manipulation of different segments of the PICA. E and F: Different trajectories in the operative view. G and H: Different trajectories from the inferior anatomical perspective. Copyright UCSF Skull Base and Cerebrovascular Laboratory. Published with permission. Figure is available in color online only.

prone position needed for accessing the inferior cerebellar peduncle are not required for exposing the LR through the tonsillobiventral approach, because there is no need to look "upward" to see the peduncle. Another difference between the supratonsillar approach to the inferior cerebellar peduncle and the tonsillobiventral approach to the LR is C-1 laminectomy. A C-1 laminectomy may be necessary to access the inferior cerebellar peduncle by providing a steeper angle of attack, but an inferolateral trajectory to reach the LR is not hindered by the lamina of $\mathrm{C}-1$ and therefore does not require a $\mathrm{C}-1$ laminectomy (Fig. 2F). 

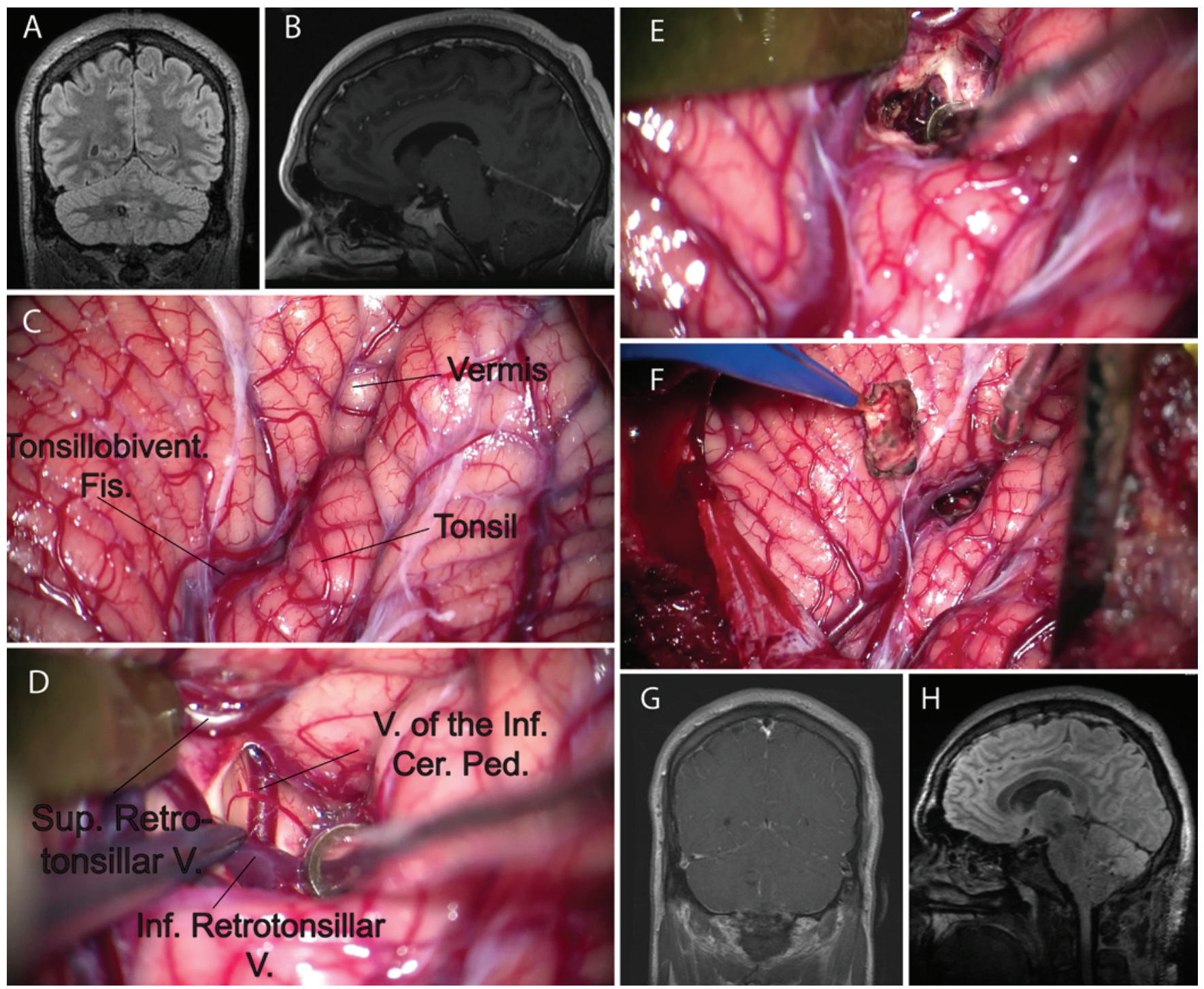

FIG. 3. Coronal FLAIR (A) and parasagittal contrast-enhanced T1-weighted (B) MR images obtained in a 24-year-old man with headaches after weight lifting showing a mixed-intensity lesion with a hypointense hemosiderin ring compatible with a cavernous malformation. After a suboccipital craniotomy in the prone position, the tonsillobiventral fissure was exposed (C) and opened (D) to expose the LR following the PICA branches, and also following the retrotonsillar and supratonsillar veins. Once the supratonsillar recess was opened, the hemosiderin staining on the cerebellar surface was identified, a small trajectory was made through the overlying cortex, and the malformation was immediately found. The malformation was dissected circumferentially and removed en bloc ( $\mathbf{E}$ and $\mathbf{F})$. Postoperative coronal $(\mathbf{G})$ and sagittal $(\mathbf{H})$ contrast-enhanced T1-weighted MR images show complete removal of the lesion. Cer. = cerebellar; Fis. = fissure; Inf. = inferior; Ped. = peduncle; Sup. = superior. V. = vein. Figure is available in color online only.

In our previous work, we stated that after reaching the inferior cerebellar peduncle "no additional subarachnoid dissection is possible."7 However, an inferolateral trajectory toward the LR allows subarachnoid dissection all the way to the cerebellomedullary cistern (Fig. 4 left). The most lateral limits of the LR can be accessed completely via the tonsillobiventral approach, which is a substantial improvement over traditional approaches (Fig. 1D, E, and $\mathrm{G})$. The results of our analysis show that opening the tonsillobiventral fissure allows access to the choroid plexus of the cerebellopontine cistern (Fig. 1A). Such exposure could not be easily obtained via the tCMF approach without excessive retraction of the tonsil (Figs. 1D and 4 right) ${ }^{4}$

Using a superolateral trajectory, the tonsillobiventral approach may also be used to access intraaxial cerebellar lesions (e.g., inferior cerebellar peduncle). ${ }^{7}$ However, exposure and resection of lesions within the LR do not involve traversing neural tissue, because following the arachnoid planes inside the tonsillobiventral fissure will directly expose the LR. Navigation planning and intraoperative guidance can help in selecting the proper trajectory to reach intracerebellar lesions, especially when invasion of the white matter is present. 

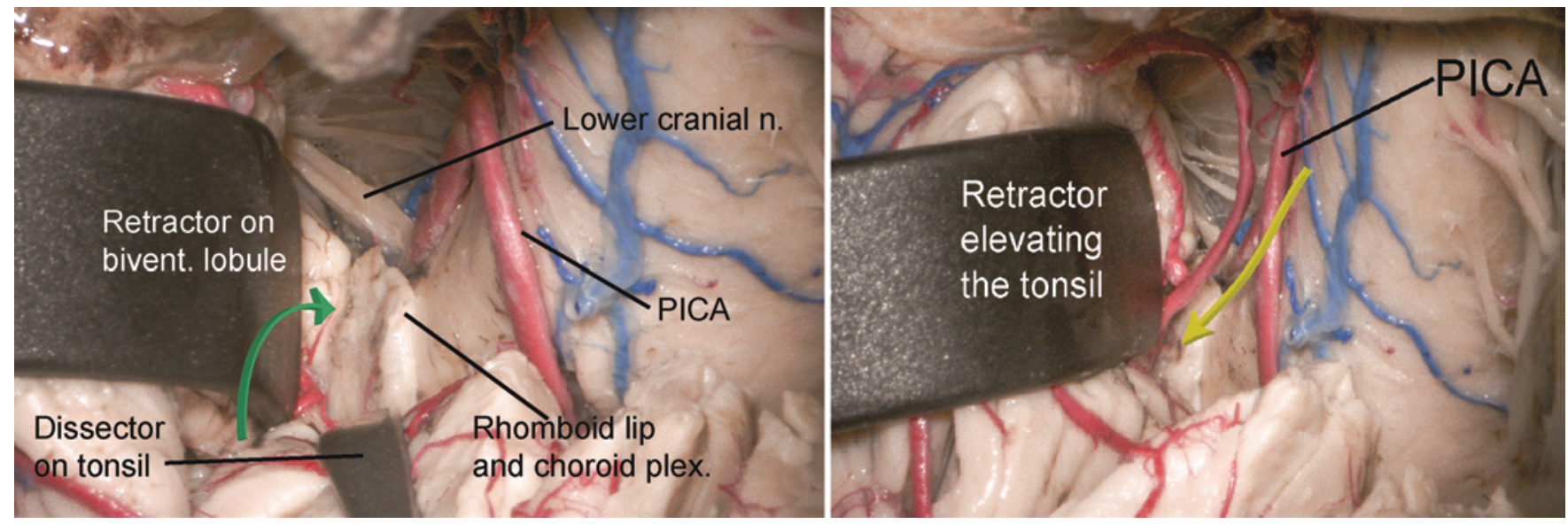

FIG. 4. Comparison of the extent of exposure of the LR between the telovelar (left) and supratonsillar (right) approaches on the right side of a cadaveric head. The green arrow and the yellow arrow designate the approaches. bivent. = biventral; $n .=$ nerve. Figure is available in color online only.

Avoidance of the telovelar segment of the PICA is another advantage of the tonsillobiventral approach. During the tCMF approach, the distal cisternal segments of PICA (i.e., tonsillomedullary $\left[\mathrm{P}_{3}\right]$ and proximal telovelotonsillar $\left.\left[\mathrm{P}_{4}\right]\right)$ are exposed and require manipulation..$^{10,14}$ These segments give rise to most medullary perforator arteries along the course of the PICA. ${ }^{13}$ Also, after opening of the LR, the PICA and its minute perforator branches remain within the working corridor during resection of the LR lesion. However, the tonsillobiventral approach exposes and follows the distal telovelotonsillar (distal $\mathrm{P}_{4}$ ) segment of the PICA to reach the LR (Fig. 1F). The distal $\mathrm{P}_{4}$ segment has fewer or no medullary perforators and therefore entails less risk of brainstem infarction. Nevertheless, it should be kept in mind that the tonsillobiventral approach does not eliminate the risk to PICA branches, although it carries less risk to brainstem perforators compared with the tCMF approach.

\section{Surgical Landmarks of the Tonsillobiventral Approach}

Although we did not find much variation in the specimens regarding landmarks for identification of the tonsillobiventral fissure, surgical identification of the tonsillobiventral fissure may not be straightforward. The tonsillar folia are not parallel to the cerebellar folia of the biventral lobule, and their intersection provides a reliable landmark to recognize the tonsillobiventral fissure during subarachnoid dissection. ${ }^{7}$ Also, the cortical branches of the PICA (at least 1 of the hemispheric branches) emerge from the tonsillobiventral fissure and may be used as a landmark for identifying the fissure. ${ }^{7,9,10,13}$ The purpose of this study was to assess the feasibility of the tonsillobiventral approach to access the LR. Although in the 6 specimens in our study we found that the course of the PICA was predictable and related strongly to the tonsillobiventral fissure, this relation could be further examined using a larger sample.

As with tonsillar resection, ${ }^{4}$ the dentate nucleus and the inferior cerebellar peduncle are at risk for damage when using a tonsillobiventral fissure trajectory. ${ }^{7}, 18$ The inferior cerebellar peduncle arches over the LR from posterior to anterior. Also, this structure forms a thin layer of white matter between the LR and the dentate nucleus (Fig. 5). Opening the arachnoid trabeculae requires utmost attention to identify the inferior cerebellar peduncle and deeper cerebellar nuclei. Because splitting the tonsillobiventral fissure provides a direct trajectory to the LR, addressing lesions concealed within the limits of the LR should not require removal of neural tissue.

Splitting the tonsillobiventral fissure can be technically challenging. Distal branches of the PICA and prominent supra- and retrotonsillar veins inside the fissure need to be identified and protected early during the subarachnoid dissection. Also, the fissure could be adherent and this may put distal PICA segments at risk. ${ }^{7}$ However, the technical difficulty of splitting the tonsillobiventral fissure may be overcome through correct patient positioning, meticulous microsurgical dissection, and protection of the pial planes that define the borders of the tonsillobiventral fissure. Table 1 summarizes the features of the tonsillobiventral approach compared with the tCMF approach.

\section{Conclusions}

The tonsillobiventral fissure approach, or supratonsillar approach, can be used to access the LR, even its most

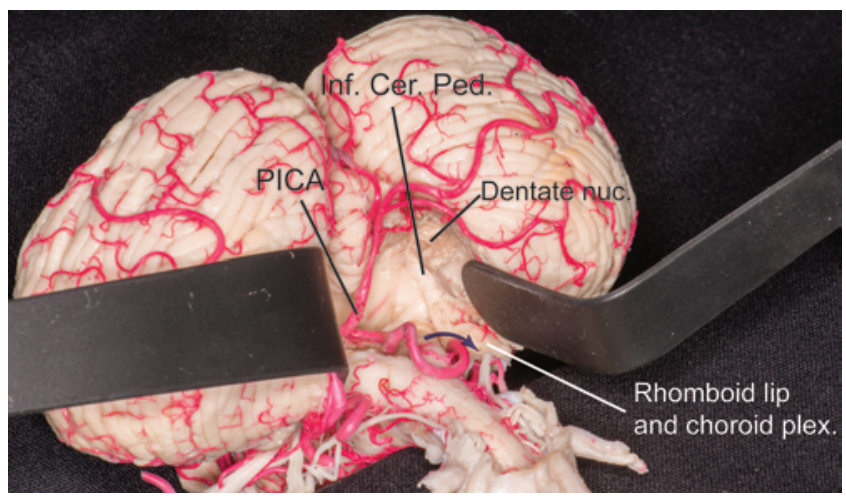

FIG. 5. Depiction of the right LR. The fibers of the inferior cerebellar peduncle (partially resected) pass in close relationship to the dentate nucleus. nuc., nucleus. Figure is available in color online only. 
TABLE 1. Comparison of different features of the tonsillobiventral and tCMF approaches

\begin{tabular}{lccccc}
\hline Approach & $\begin{array}{c}\text { Tonsillar } \\
\text { Retraction }\end{array}$ & $\begin{array}{c}\text { Surgical } \\
\text { Trajectory }\end{array}$ & Structures at Risk & $\begin{array}{c}\text { Identification } \\
\text { of Corridor }\end{array}$ & $\begin{array}{c}\text { Exposure of Most } \\
\text { Lateral Extent of LR }\end{array}$ \\
\hline Tonsillobiventral & Less & Vertical & Inferior cerebellar peduncle & Difficult & Easily possible \\
\hline tCMF & More & Parallel & PICA perforators & Easy & Difficult \\
\hline
\end{tabular}

* Trajectory relative to brainstem axis.

lateral extent. Compared with transvermian and tCMF approaches, this approach has a more favorable trajectory that uses gravity retraction to naturally unfold the tonsillobiventral fissure, and it reduces fixed cerebellar retraction. Also, vital brainstem perforator arteries originating from the $\mathrm{P}_{3}$ and proximal $\mathrm{P}_{4}$ segments of the PICA are avoided during the tonsillobiventral fissure approach. Limitations of this approach include possible damage to the distal PICA branches, the inferior cerebellar peduncle, and the dentate nucleus, and difficulty in identifying the tonsillobiventral fissure. However, anatomical landmarks are reliable and meticulous microsurgical dissection of arachnoid planes inside the fissure with navigation guidance maximizes safety. This approach has been a useful addition to the surgical armamentarium for cavernous malformations inside the inferior cerebellar peduncle and those in the vicinity of LR, and may have additional application to vascular and tumor lesions in the LR.

\section{Acknowledgments}

We acknowledge the artist Kenneth X. Probst for the artwork included in this report. We express our gratitude to the body donors and their families, who through their altruism contributed to making this project possible.

\section{References}

1. Benet A, Rincon-Torroella J, Lawton MT, González Sánchez $\mathrm{JJ}$ : Novel embalming solution for neurosurgical simulation in cadavers. J Neurosurg 120:1229-1237, 2014

2. Deshmukh VR, Figueiredo EG, Deshmukh P, Crawford NR, Preul MC, Spetzler RF: Quantification and comparison of telovelar and transvermian approaches to the fourth ventricle. Neurosurgery 58 (4 Suppl 2):ONS-202-ONS-207, 2006

3. Jean WC, Abdel Aziz KM, Keller JT, van Loveren HR: Subtonsillar approach to the foramen of Luschka: an anatomic and clinical study. Neurosurgery 52:860-866, 2003

4. Jittapiromsak P, Sabuncuoglu H, Deshmukh P, Spetzler RF, Preul MC: Accessing the recesses of the fourth ventricle: comparison of tonsillar retraction and resection in the telovelar approach. Neurosurgery 66 (3 Suppl Operative):30-40, 2010

5. Kachhara R, Raje P, Pauranik A: Schwannoma originating in lateral recess of the fourth ventricle. Asian J Neurosurg 7:151-153, 2012

6. Kawashima M, Matsushima T, Nakahara Y, Takase Y, Masuoka J, Ohata K: Trans-cerebellomedullary fissure approach with special reference to lateral route. Neurosurg Rev 32:457-464, 2009

7. Lawton MT, Quiñones-Hinojosa A, Jun P: The supratonsillar approach to the inferior cerebellar peduncle: anatomy, surgical technique, and clinical application to cavernous malformations. Neurosurgery 59 (4 Suppl 2):ONS244-ONS252, 2006

8. Lee CC, Lin CF, Yang TF, Hsu SP, Chen HH, Chen SC, et al: Telovelar approach for choroid plexus papilloma in the foramen of Luschka: a safe way using a neuromonitor. Clin Neurol Neurosurg 114:249-253, 2012

9. Lister JR, Rhoton AL Jr, Matsushima T, Peace DA: Microsurgical anatomy of the posterior inferior cerebellar artery. Neurosurgery 10:170-199, 1982

10. Mussi AC, Rhoton AL Jr: Telovelar approach to the fourth ventricle: microsurgical anatomy. J Neurosurg 92:812-823, 2000

11. Oliver J, Middleditch A: Functional Anatomy of the Spine. Oxford: Butterworth-Heinemann, 1991

12. Rekate HL, Grubb RL, Aram DM, Hahn JF, Ratcheson RA: Muteness of cerebellar origin. Arch Neurol 42:697-698, 1985

13. Rhoton AL Jr: The cerebellar arteries. Neurosurgery 47 (3 Suppl):S29-S68, 2000

14. Rodríguez-Hernández A, Rhoton AL Jr, Lawton MT: Segmental anatomy of cerebellar arteries: a proposed nomenclature. Laboratory investigation. J Neurosurg 115:387-397, 2011

15. Strauss C, Fahlbusch R: Anatomical aspects for surgery within the floor of the IVth ventricle. Zentralbl Neurochir 58:7-12, 1997

16. Tanriover N, Ulm AJ, Rhoton AL Jr, Yasuda A: Comparison of the transvermian and telovelar approaches to the fourth ventricle. J Neurosurg 101:484-498, 2004

17. Tomasello F, Conti A, Cardali S, La Torre D, Angileri FF: Telovelar approach to fourth ventricle tumors: highlights and limitations. World Neurosurg 83:1141-1147, 2015

18. Yaşargil MG, Abdulrauf SI: Comment on "The supratonsillar approach to the inferior cerebellar peduncle: anatomy, surgical technique, and clinical application to cavernous malformations." Neurosurgery 59 (4 Suppl 2):ONS251, 2006

19. Ziyal IM, Sekhar LN, Salas E: Subtonsillar-transcerebellomedullary approach to lesions involving the fourth ventricle, the cerebellomedullary fissure and the lateral brainstem. Br J Neurosurg 13:276-284, 1999

\section{Disclosures}

The authors report no conflict of interest concerning the materials or methods used in this study or the findings specified in this paper.

\section{Author Contributions}

Conception and design: Benet, Tayebi Meybodi, Lawton. Acquisition of data: Benet, Tayebi Meybodi, Tabani. Analysis and interpretation of data: all authors. Drafting the article: Benet, Tayebi Meybodi, Tabani. Critically revising the article: Benet, Lawton, Tabani. Reviewed submitted version of manuscript: all authors. Approved the final version of the manuscript on behalf of all authors: Benet. Statistical analysis: Benet, Tayebi Meybodi, Lawton. Administrative/technical/material support: Benet, Lawton. Study supervision: Benet, Lawton.

\section{Correspondence}

Arnau Benet, Department of Neurosurgery, Rm. M-779, University of California, San Francisco, 505 Parnassus Ave., San Francisco, CA 94143. email: arnau.benet@ucsf.edu. 